\title{
Activation of AMP-activated kinase modulates sensitivity of glioma cells against epidermal growth factor receptor inhibition
}

\author{
INES HARTEL $^{1}$, MICHAEL RONELLENFITSCH ${ }^{1}$, CHRISTINA WANKA $^{1}$, STEFAN WOLKING $^{2}$, \\ JOACHIM P. STEINBACH ${ }^{1,3}$ and JOHANNES RIEGER ${ }^{1}$ \\ ${ }^{1}$ Dr. Senckenberg Institute of Neurooncology, Goethe University Frankfurt, D-60528 Frankfurt; \\ ${ }^{2}$ Department of Neurology and Epileptology, Hertie Institute for Clinical Brain Research, D-72076 Tübingen; \\ ${ }^{3}$ German Cancer Consortium (DKTK) and German Cancer Research Center (DKFZ), Heidelberg, Germany
}

Received December 15, 2015; Accepted January 20, 2016

DOI: 10.3892/ijo.2016.3498

\begin{abstract}
The epidermal growth factor (EGFR) pathway is frequently activated in glioblastoma but the clinical efficacy of EGFR inhibitors in malignant glioma has been disappointing. The reasons for the failure of the mechanisms of resistance of these inhibitors are unclear, but may involve factors of the tumor microenvironment such as limited glucose availability and hypoxia. It was therefore examined whether glucose and oxygen influenced the response of glioma cells to EGFR inhibition. Decreased levels of glucose and oxygen led to resistance against the EGFR inhibitor PD153035, whereas high glucose amounts and normoxia sensitised glioma cells towards the inhibitor. Low levels of glucose and oxygen stimulated AMP-activated kinase (AMPK) in glioma cells. 2DG, an inhibitor of glycolysis, and the AMPK activator A769662 reduced glucose consumption, induced phosphorylation of AMPK and mimicked the effects of low glucose availability on the toxicity of PD153035. Similarly, 2DG reduced toxicity of imatinib in K562 leukemia cells. In contrast, inhibition of AMPK by compound C or by short-hairpin (sh)-mediated gene suppression increased cell death induced by the EGFR inhibitor and reverted the protective effects of $2 \mathrm{DG}$ and
\end{abstract}

Correspondence to: Dr Johannes Rieger, Dr. Senckenberg Institute of Neurooncology, Goethe University Frankfurt, Schleusenweg 2-16, D-60528 Frankfurt, Germany

E-mail: johannes.rieger@med.uni-frankfurt.de

Abbreviations: 2DG, 2-deoxy-D-glucose; ACC, acetyl-CoAcarboxylase; AMPK, AMP-activated kinase; EGFR, epidermal growth factor receptor; ERK1/2, extracellular signal-regulated kinase $1 / 2$; mTOR, mammalian target of rapamycin; PI, propidium iodide; PKB, protein kinase B; ROS, reactive oxygen species; shRNA, short-hairpin RNA

Key words: glucose, glioma, AMP-activated kinase, epidermal growth factor receptor, hypoxia, 2-deoxy-D-glucose
A769662. In conclusion, cytotoxicity of EGFR inhibition can be diminished by AMPK activation in glioma cells. These results may provide one explanation for the low activity of EGFR inhibitors in clinical trials and suggest antagonism of AMPK or of AMPK-regulated metabolic alterations as a promising approach to enhance their therapeutic efficacy.

\section{Introduction}

Despite multimodal treatment with surgery, radiotherapy and chemotherapy, glioblastoma is still associated with poor prognosis (1). Reasons why glioma are refractory to current conventional therapeutic approaches involve their infiltrative growth patterns preventing radical resection and cell autonomous anti-apoptotic mechanisms. These properties could be mediated at least partly by the epidermal growth factor receptor (EGFR) which is among the most overexpressed and/or mutated protein in gliomas (2). Therefore, targeting the EGFR pathway could be a promising therapeutic approach. However, clinical results exploring EGFR inhibitors against glioma have been disappointing thus far (3), and the causes for the lack of clinical activity of EGFR inhibitors are only incompletely understood.

A factor which might influence the efficacy of EGFR inhibitors could be the tumor microenvironment characterised by areas of nutrient and oxygen deprivation (4). We therefore previously investigated the effects of EGFR inhibition under severe hypoxia and found that hypoxia-induced cell death in glioma cells was reduced by an EGFR inhibitor, at least partly by reduced energy consumption (5). This energy conserving mechanism included reduced glucose consumption, resulting in slower ATP depletion and decreased cytochrome $c$ release (5). Further analyses showed that inhibition of mammalian target of rapamycin (mTOR), a downstream effector of growth factor receptors, similarly reduced energy demands and cell death under hypoxia (6), underlining the importance of mTOR as a central regulator of cellular energetics (7). As hypoxia is present in large areas of glioma before treatment and probably is even more severe after anti-angiogenic treatments (8), these observations might be an explanation for 
the low efficacy of EGFR inhibitors in glioma patients. On the contrary, EGFR inhibition sensitised glioma cells to death ligands under normoxic conditions (9) indicating that glioma cells are not completely refractory against anti-apoptotic effects of EGFR inhibition. However, how exactly metabolic conditions mediate these different effects is unclear, but understanding the mechanism that determine the outcome of EGFR inhibition is important to improve the efficacy of growth factor receptor-inhibiting therapies. In this study, we therefore investigated in more detail how glucose and oxygen influence the effect of EGFR blockade in glioma cells and found that the AMP-activated kinase (AMPK) increases resistance against EGFR inhibition.

\section{Materials and methods}

Cell culture. LNT-229 glioma cells were described previously (10). U87MG, the leukemia cell line K562 as well as the breast cancer cell line MDA-MB-435 were obtained from the ATCC (Wesel, Germany). Cells were cultured in Dulbecco's modified Eagle's medium (DMEM, Sigma-Aldrich, Taufkirchen, Germany) containing 10\% fetal calf serum (FCS), 2 mM glutamine, $100 \mathrm{IU} / \mathrm{ml}$ penicillin, and $100 \mathrm{mg} / \mathrm{ml}$ streptomycin. For experimental procedures involving limited glucose concentrations, we used Dulbecco's modified Eagle's glucose-free medium (Life Technologies, Darmstadt, Germany) without FCS, and glucose was added to obtain the required concentration. Cells were seeded at a density of 80,000 cells/well in 24-well plates for FACS analysis and 1,500,000 cells/well in $10-\mathrm{cm}$ dishes for protein extraction if not otherwise specified. LNT-229 cells stably transfected with wild-type-EGFR (pLERNL) were described previously (9).

Primary cell culture. The glioblastoma derived cell line MNOF132 was kindly provided by Stefan Momma (Edinger Institute, Goethe-University Frankfurt). Cells were cultured in DMEM-F12 Medium containing $20 \mathrm{ng} / \mathrm{ml}$ of epidermal growth factor (EGF, ReliaTech, Wolfenbüttel, Germany) and human basic fibroblast growth factor-2 (bFGF-2, ReliaTech, Wolfenbüttel, Germany) as well as $20 \%$ BIT admixture 100 supplement (Pelo Biotech, Planegg/Martinsried, Germany). During cell culture EGF and bFGF-2 were added to the cells twice per week.

Reagents and treatments. The following reagents were used: 2-deoxy-D-glucose (2DG) (Sigma-Aldrich), PD153035 hydrochloride (Biozol, Eching, Germany), A769662 (RnD Systems, Minneapolis, MN, USA), zVAD-fmk (Bachem, Weil am Rhein, Germany) and compound C (Sigma-Aldrich). Cells were seeded and, after $24 \mathrm{~h}$, treated with either PD153035 $(10 \mu \mathrm{M}), \mathrm{A} 769662$ $(100 \mu \mathrm{M}), 2 \mathrm{DG}(10 \mathrm{mM})$, compound $\mathrm{C}(5 \mu \mathrm{M})$ or zVAD $(100 \mu \mathrm{M})$ for the indicated time-points in Dulbecco's modified Eagle's glucose-free medium (Life Technologies) in which glucose was added as required. For treatment of MDA-MB-435 and of K562 cells, lapatinib $(10 \mu \mathrm{M})$ was obtained from Sequoia laboratory (Berkshire, UK), and imatinib $(10 \mu \mathrm{M})$ was obtained from Enzo Life Sciences (Lörrach, Germany).

Lentiviral transduction. For AMPK double knockout LNT229 cells were transfected with pLKO.1 short hairpin RNA
(shRNA) plasmids from Sigma-Aldrich (TRCN0000196482, TRCN0000355741). The non-targeting plamids were from Addgene (\#1864, \#10905). Production of lentivirus and transfection of the cells were done as previously described (11). For selection $2 \mu \mathrm{g} / \mathrm{ml}$ puromycin and $800 \mu \mathrm{g} / \mathrm{ml}$ hygromycin was used.

Induction of hypoxia. To induce $0.1 \% \mathrm{O}_{2}$, cells were incubated in GasPak pouches for anaerobic culture (Becton-Dickinson, Heidelberg, Germany) (10). Moderate hypoxia $\left(1 \% \mathrm{O}_{2}\right)$ was induced in a $\mathrm{CO}_{2}$-incubator (Binder, Germany) (12).

Cell death analysis. Cells were seeded at 80,000 cells per well in 24-well-plates. After $24 \mathrm{~h}$, medium was removed, cells were washed, and incubated with the appropriate reagents. For the detection of cell death, cells were stained with propidium iodide (PI) and analysed by FACS as previously described (12). Experiments were performed in triplicates and presented as mean $\pm \mathrm{SD}$.

Cell cycle analysis. To assess cell cycle distribution, cells were treated as indicated, fixed in $70 \%$ ice-cold ethanol and incubated gently vortexing for $45 \mathrm{~min}$. After centrifugation, the pellet was resuspended in $0.1 \mathrm{ml} \mathrm{RNaseA}(20 \mu \mathrm{g} / \mathrm{ml})$ and incubated for $5 \mathrm{~min}$ before $400 \mu \mathrm{l}$ PI $(50 \mu \mathrm{g} / \mathrm{ml})$ was added. After a second incubation step for $30 \mathrm{~min}$, cells were filtered through $50 \mu \mathrm{l}$ filcons before starting FACS analysis. Cells with a sub-G1 DNA content were considered as dead cells.

Caspase activation assay. Caspase activation was assessed with caspase activity assay (Roche Diagnostic, Mannheim, Germany) according to the manufacturer's protocol.

Flow cytometry analysis. Cells were harvested with accutase, and 500,000 cells per sample were washed twice with FACS buffer (PBS with 2\% FCS) and centrifuged by $1200 \mathrm{x} \mathrm{g}$ for $3 \mathrm{~min}$. Thereafter, the isotype (M5534, Sigma-Aldrich, Steinheim, Germany) or the anti-EGFR antibody (Alexa Fluor 647 (sc-101 AF647), R-1, Santa Cruz, USA) was added, the pellet was resuspended, and the cells were incubated on ice for $1 \mathrm{~h}$. Subsequently, cells were washed twice and kept on ice until the measurement started. Cells were analysed by flow cytometry in a BD Canto II using the PE-channel. Specific fluorescence index (SFI) was calculated as median fluorescence intensity of specific antibody/median fluorescence index of isotype antibody.

Immunoblot analysis. Cells were seeded in $10-\mathrm{cm}$ plates and treated as indicated. Thereafter, the cells were washed with ice cold phosphate-buffered saline (PBS) and lysed in lysis buffer (50 mM Tris-HCl pH 8.0, 120 mM NaCl, 5 mM EDTA, $0.5 \%$ Nonidet P-40) containing protease inhibitors (Roche Applied Science, Mannheim, Germany). Cellular lysates were prepared as described (13) and subjected to sodium dodecyl sulfate-polyacrylamide gel electrophoresis (SDS-PAGE). Membranes were probed with antibodies as listed below. The chemiluminescence solution used for detection was composed of $1 \mathrm{ml}$ of solution A (200 ml of $0.1 \mathrm{M}$ Tris- $\mathrm{HCl} \mathrm{pH} \mathrm{8.6,} 50 \mathrm{mg}$ of luminol), $100 \mu \mathrm{l}$ of solution B [11 mg of p-hydroxycoumarin acid, $10 \mathrm{ml}$ dimethyl sulfoxide (DMSO)], and $0.3 \mu \mathrm{l}$ of $\mathrm{H}_{2} \mathrm{O}_{2}$ 
(30\%). Antibodies against the following antigens were used: rabbit anti-phospho AMPK $\alpha$ Thr172, rabbit anti-AMPK $\alpha$, rabbit anti-pACC Ser79 (D7D11), rabbit anti-ACC and rabbit Pathscan Muliplex Western Cocktail I (phospho-p90RSK, phospho-Akt, phospho-Erk1/2, phospho-S6 ribosomal protein) were obtained from Cell Signaling Technologies (Danvers, MA, USA), and mouse anti-GAPDH (MAB374) from Chemicon (Nuernberg, Germany). Secondary antibodies and rabbit anti-EGFR were purchased from Santa Cruz Biotechnology.

RNA extraction and quantitative RT-PCR (qRT-PCR). Total RNA was extracted using TRIzol and PureLink ${ }^{\circledR}$ RNA Mini kit (Life Technologies Ambion ${ }^{\circledR}$ ). The Vilo cDNA synthesis kit (Life Technologies Invitrogen ${ }^{\mathrm{TM}}$ ) was used for the synthesis of first strand cDNA for $10 \mathrm{~min}$ at $25^{\circ} \mathrm{C}$ and $2 \mathrm{~h}$ at $42^{\circ} \mathrm{C}$. Following this, the enzyme was inactivated at $85^{\circ} \mathrm{C}$ for $10 \mathrm{~min}$. PCR was performed using $15 \mu \mathrm{g}$ RNA and absolute Blue Q-PCR master mix with SYBR Green + fluorescein (Thermo Fisher Scientific, Hamburg, Germany). The reactions were cycled 30 times $\left[50^{\circ} \mathrm{C}\right.$ for $2 \mathrm{~min}$ and $95^{\circ} \mathrm{C}$ for $10 \mathrm{~min}\left(94^{\circ} \mathrm{C}\right.$ for $15 \mathrm{sec}, 58-60^{\circ} \mathrm{C}$ for $1 \mathrm{~min}$, and $72^{\circ} \mathrm{C}$ for $\left.1 \mathrm{~min}\right) \times 30$ cycles]. The following primer pairs were used: AMPK $\alpha 1$ forward 5'-AGAAGCAG AAACACGACGGG-3', AMPK $\alpha 1$ reverse 5'-GCGGATTTT TCCTACCACATCA-3', AMPK $\alpha 2$ forward 5'-CGGCTCTTT CAGCAGATTCTGT-3', AMPK $\alpha 2$ reverse 5'-ATCGGCTAT CTTGGCATTCATG-3', SDHA forward 5'-TGGGAACA AGAGGGCATCTG-3' and SDHA reverse 5'-CCACCACTGC ATCAAATTCATG-3'. Cycle threshold $(\mathrm{Ct})$ values were normalized for amplification of the SDHA RNA, and the data were analysed using the Vandesompele method as described (13).

Glucose/lactate measurements. To determine glucose consumption and lactate production, the supernatant was collected and cells were removed by centrifugation. Glucose and lactate concentrations were measured using the biochemistry analyser Hitachi 917.

Ethics statement. The use of the primary glioma cell line was approved by the ethics committee of the University Hospital Frankfurt (reference number 4/09).

\section{Results}

Metabolic conditions influence the effect of EGFR inhibition. To investigate whether two important metabolic determinants of the tumor microenvironment, glucose and oxygen, modulate the toxicity of EGFR inhibition, glioma cells were exposed to different concentrations of glucose and oxygen in the absence or presence of an EGFR inhibitor (PD153035), and cell death was analysed (Fig. 1A and C). Hypoxia-induced cell death was enhanced at low glucose concentrations, suggesting that glucose availability is a limiting factor for survival of glioma cells under hypoxia (Fig. 1A and $\mathrm{C}$ right panel; Fig. 1B). As previously shown, PD153035 inhibited glioma cell death at hypoxia and low glucose levels (5). Under normoxia, however, PD153035 induced cell death, and, surprisingly, this effect was more pronounced if large amounts of glucose were available (Fig. 1A and C left panel; Fig. 1B). Increased toxicity under abundant glucose was also present under hypoxia in LNT-229 cells, but less pronounced (Fig. 1A). Fig. 1D summarises the effect of EGFR inhibition under the different metabolic conditions.

EGFR inhibition induces caspase-dependent cell death. To characterise cell death induced by PD153035, we asked whether caspases were involved. As shown in Fig. 2A, the EGFR inhibitor induced caspase activation, and this effect again was suppressed by low glucose concentrations. The pan-caspase inhibitor zVAD-FMK strongly suppressed caspase activation and blocked PD153035-mediated cell death, suggesting that cytotoxicity of the EGFR inhibitor was caspase-dependent (Fig. 2A and B). Additionally, the PD153035-induced increase in sub-G1 fraction indicative of nuclear fragmentation during apoptosis was inhibited by zVAD-FMK (Fig. 2C). Together, these experiments suggest that the EGFR inhibitor PD153035 induced caspase-dependent cell death.

EGF receptor expression and downstream signaling. Having established that low glucose levels inhibit cytotoxicity of the EGFR inhibitor, we asked whether reduced expression of EGFR under low glucose conditions might be responsible for the observed effect. However, immunoblot analysis did not reveal any modulation in EGFR expression under different glucose concentrations (Fig. 3A). Similarly, surface expression of EGFR remained unaltered by different glucose concentrations (Fig. 3B). We further investigated whether different glucose concentrations modulated response of downstream effectors to the EGFR inhibitor. As expected, PD153035 diminished the phosphorylation of extracellular signal-regulated kinase 1/2 (ERK1/2), protein kinase B (PKB)/Akt and S6 kinase, but this effect was unaffected by the different experimental conditions (Fig. 3C). Together, the reduced toxicity of EGFR inhibition under low glucose concentration could not be explained by reduced expression of EGFR or diminished pathway inhibition.

Activation of AMPK protects against EGFR inhibition. The suppression of PD153035-mediated toxicity by limited glucose availability suggested that AMP-activated protein kinase (AMPK), a major sensor for cellular energy status, might influence the outcome of EGFR inhibition. Indeed, low glucose concentrations led to phosphorylation of AMPK and its downstream target acetyl-CoA-carboxylase (ACC) (Fig. 4A). Therefore, we investigated whether activation of AMPK by two chemically unrelated activators, the glucose analogue 2-deoxy-D-glucose (2DG) and A769662, a direct activator of AMPK, modulated EGFR-inhibitor dependent cytotoxicity. As expected, these activators induced phosphorylation of AMPK and $\mathrm{ACC}$ in a similar extent (Fig. 4B). In line with the AMPK function in suppressing glycolysis, the activators significantly reduced glucose consumption and lactate production (Fig. 4C and D). Interestingly, 2DG and A769662 significantly diminished the toxicity of PD153035 suggesting that activation of AMPK indeed protected against EGFR inhibitor-induced cell death (Fig. 4E). Similar results could be observed in the U87MG cell line and in a primary glioma cell line (Fig. 5A and B). To analyse whether the antagonistic effects of AMPK activators towards tyrosine kinase inhibitors are specific for 
A

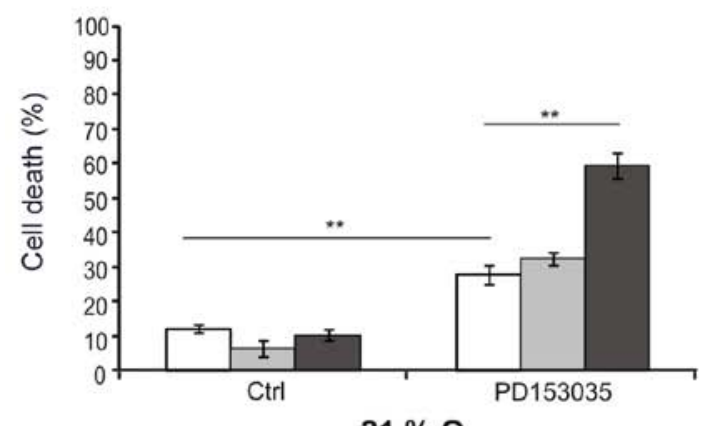

$21 \% \mathrm{O}_{2}$
LNT-229

$\square 2 \mathrm{mM} \mathrm{Glc} \quad \square 5 \mathrm{mM} \mathrm{Glc}$

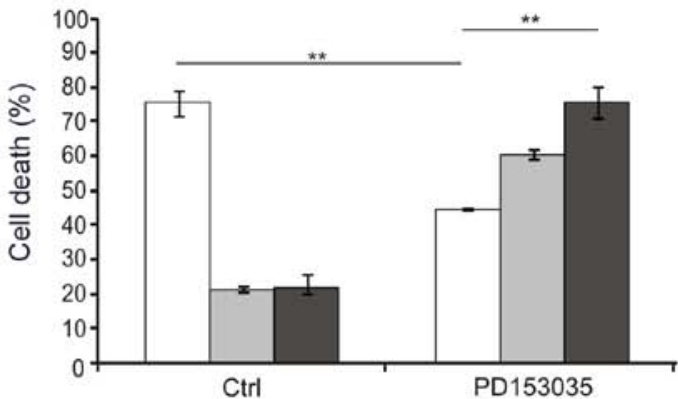

$0.1 \% \mathrm{O}_{2}$

B

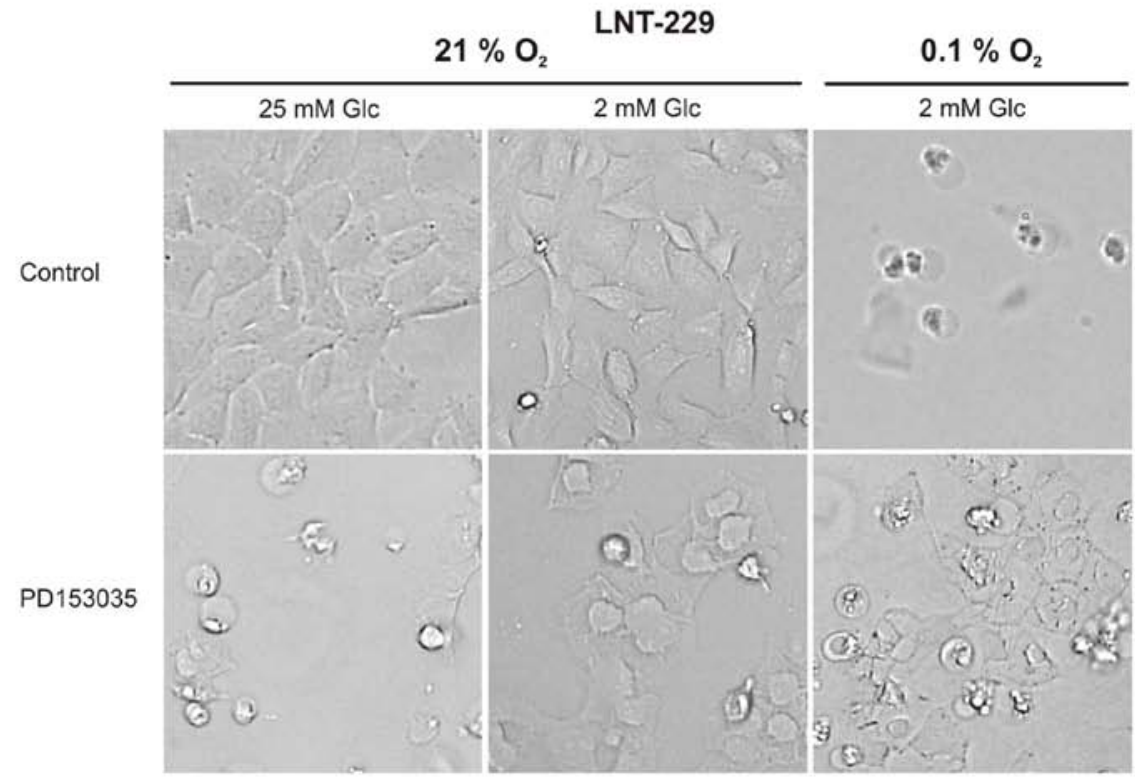

C

U87MG

$\square 2 \mathrm{mMGlc} \quad 25 \mathrm{mMGlc}$

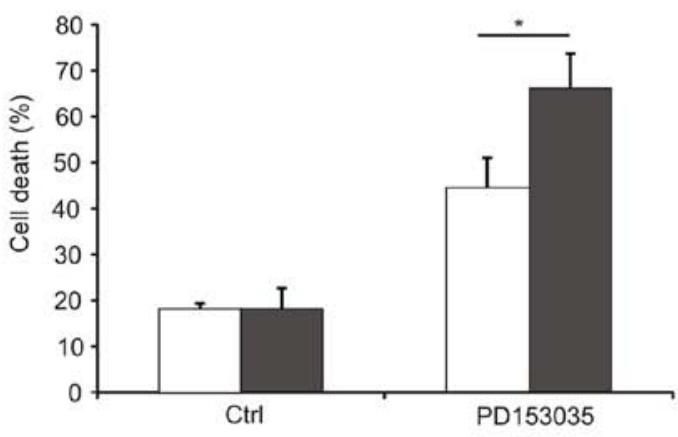

$21 \% \mathrm{O}_{2}$

D

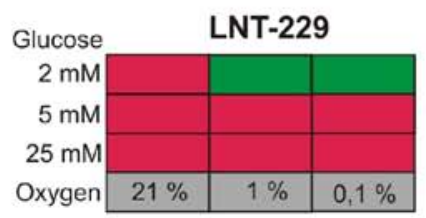

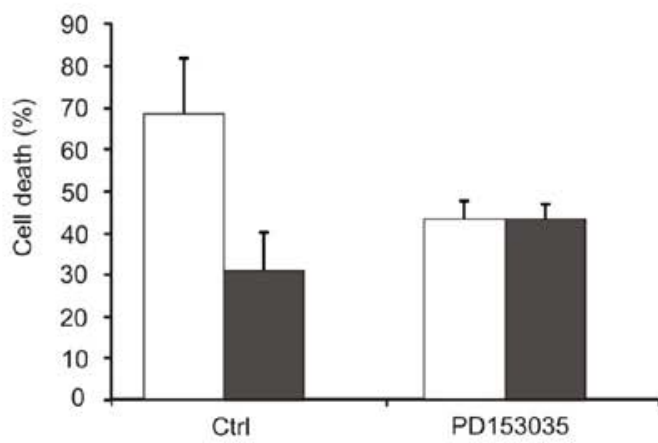

$0.1 \% \mathrm{O}_{2}$

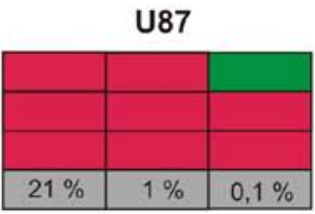

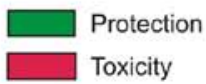

Toxicity

Figure 1. Metabolic conditions influence the cytotoxicity of EGFR inhibition. (A) LNT-229 cells were treated with the EGFR inhibitor PD153035 (10 $\mu$ M) in the presence of different glucose $(2,5$ and $25 \mathrm{mM})$ and oxygen $(21$ and $0.1 \%)$ concentrations for $48 \mathrm{~h}$, and cell death was analysed by PI staining (means \pm SD of triplicates, one experiment out of three with similar results is shown; ${ }^{* *} \mathrm{p}<0.01$, unpaired Student's t-test). (B) Microscopic analysis of the morphology of LNT-229 cells treated as described. (C) U87MG cells were treated as indicated for $72 \mathrm{~h}$, and cell death was analysed by PI FACS. (D) Schematic representation of the effect of PD153035 at different glucose and oxygen concentrations in two glioma cell lines (red, cytotoxicity; green, protection). 

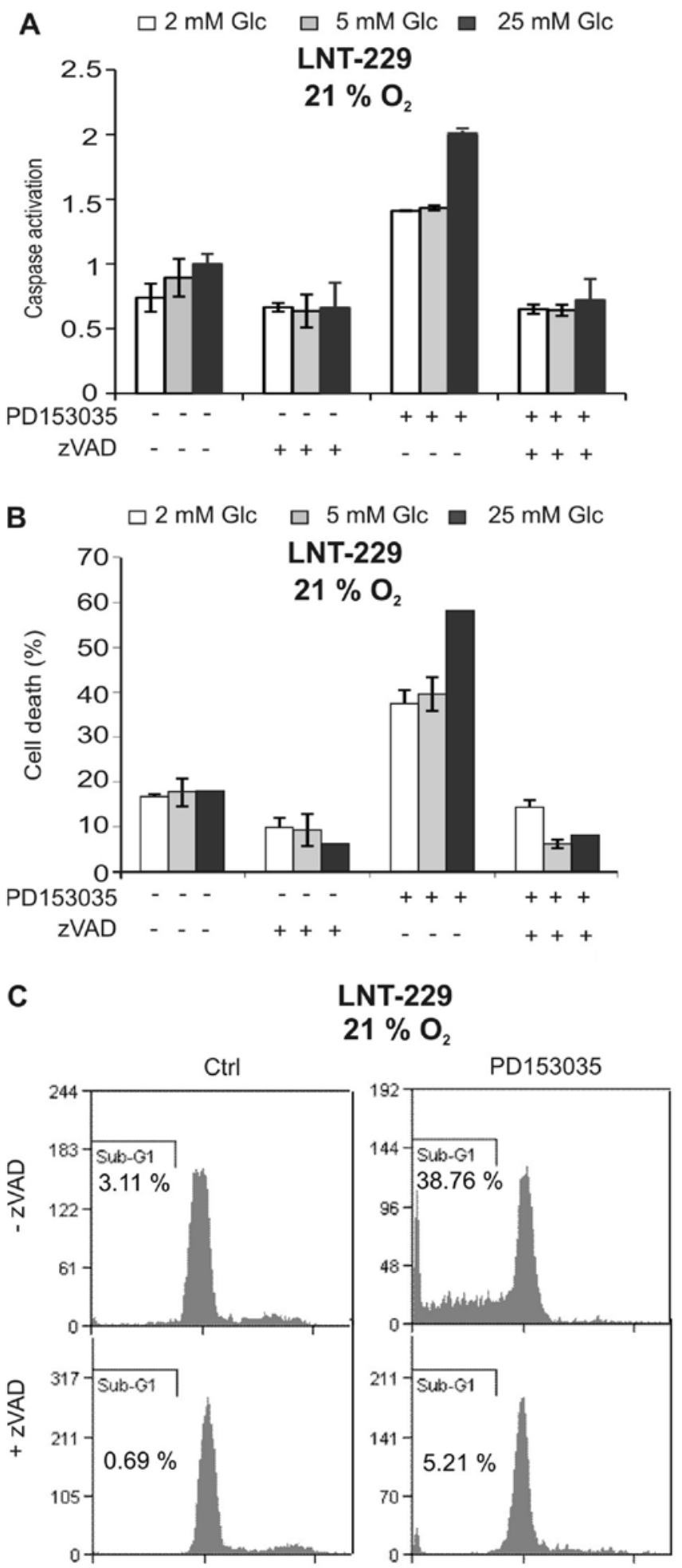

Figure 2. PD153035-induced cell death involves caspase activation. (A) LNT-229 glioma cells were treated with PD153035 either alone or in combination with zVAD-FMK $(100 \mu \mathrm{M})$ for $24 \mathrm{~h}$ under normoxia, and caspase-3/-7 activity was assessed. (B) LNT-229 cells were treated as in (A) for $48 \mathrm{~h}$, and cell death was measured by PI-FACS. (C) LNT-229 cells were treated as in (A) for $48 \mathrm{~h}$, and sub-G1 peak was analysed by cell cycle analysis (in $\mathrm{A}$ and $\mathrm{B}$, means $\pm \mathrm{SD}$ of triplicates are shown).

glioma cells, additional cellular models of tyrosine-kinaseinhibitor-induced cell death were studied. 2DG similarly reduced cytotoxicity of imatinib against K562 cells (Fig. 5C).
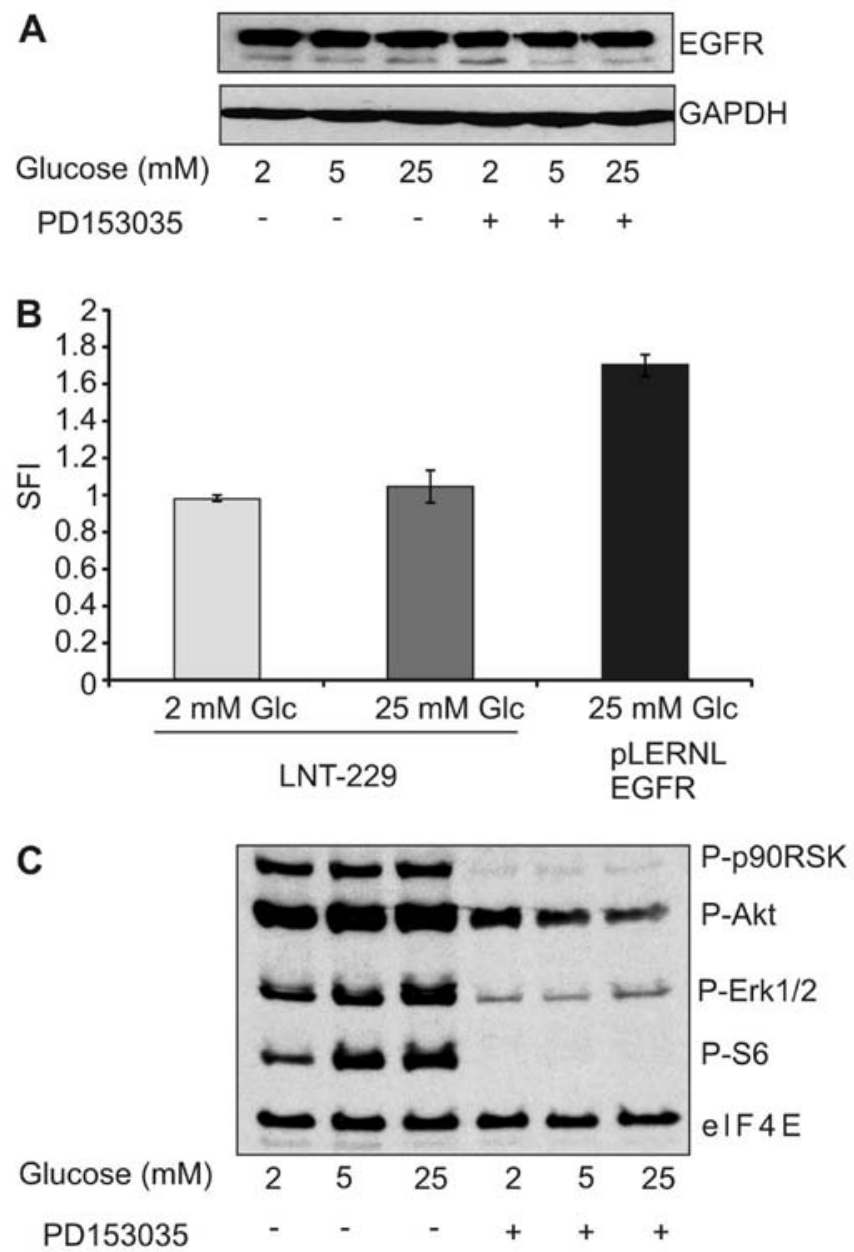

Figure 3. EGFR expression level and downstream signaling. (A) LNT-229 cells were treated as indicated for $6 \mathrm{~h}$, and EGFR expression was analysed by immunoblot analysis. GAPDH was used as a loading control. (B) LNT-229 cells were incubated at 2 and $25 \mathrm{mM}$ glucose, and surface expression of EGFR was analysed by flow cytometry. LNT-229 cells ectopically expressing EGFR (pLERNL EGFR) were used as positive control. Specific fluorescence index (SFI) was calculated as median fluorescence intensity of specific antibody/median fluorescence index of isotype antibody. (C) LNT-229 cells were incubated in different glucose concentrations in the presence or absence of PD153035 for $48 \mathrm{~h}$, and EGFR downstream signaling cascade was examined by immunoblot analysis (P-p90RSK, P-Akt, P-ERK1/2, P-S6; phosphorylated forms of p90RSK, Akt, ERK1/2, S6 ribosomal protein). eIF4E is included as a loading control.

In contrast, it sensitised MDA-MB-435 cells against cell death induction by lapatinib (Fig. 5D).

Knockdown or inhibition of AMPK sensitises against EGFR inhibition. To further confirm the role of AMPK, expression of AMPK was inhibited by lentiviral knockdown. Stable expression of the AMPK-specific shRNA significantly reduced expression of AMPK on the RNA (Fig. 6A) and protein level (Fig. 6B). Accordingly, phosphorylation of the downstream effector ACC was inhibited in these cells (Fig. 6B). Additionally, the cytotoxicity of PD153035 was higher in the AMPK-knockdown cells, and 2DG and A769662 could no longer block cell death in these cells (Fig. 6C). Similarly, pharmacological blockade of AMPK reduced phosphorylation of AMPK and ACC (Fig. 6D) and increased cell death induction by PD153035 in wild-type LNT-229 cells (Fig. 6E). 

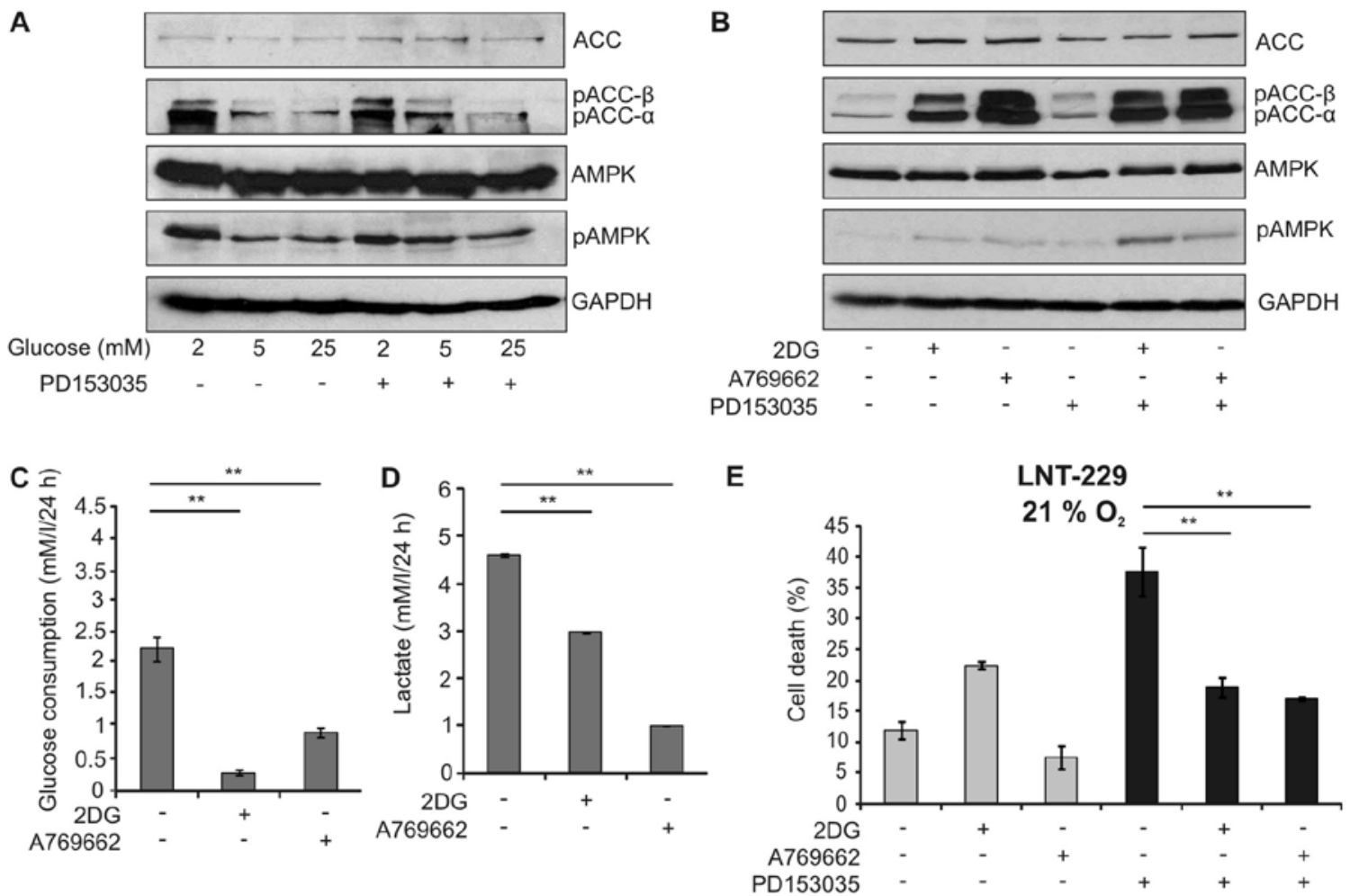

Figure 4. AMPK activation protects glioma cells from PD 153035-induced cell death. (A) LNT-229 cells were incubated at different glucose concentrations in the absence or presence of PD153035 for $6 \mathrm{~h}$, and phosphorylation of AMPK and ACC was analysed by immunoblot analysis. (B) LNT-229 cells were incubated at $25 \mathrm{mM}$ glucose in the absence or presence of 2DG $(10 \mathrm{mM})$, A769662 $(100 \mu \mathrm{M})$ and PD153035 $(10 \mu \mathrm{M})$ for $6 \mathrm{~h}$, and immunoblot analysis was carried out as in (A) (pACC, pAMPK; phosphorylated forms of ACC and AMPK). GAPDH was used as loading control. (C-E) Cells were treated as indicated for $48 \mathrm{~h}$ in medium containing $25 \mathrm{mM}$ glucose, and (C) glucose consumption, (D) lactate production and (E) cell death was analysed (shown are means \pm SD of triplicates, one experiment out of three or more independent experiments with similar results is shown; ${ }^{* *} \mathrm{p}<0.01$, unpaired Student's t- test).

A

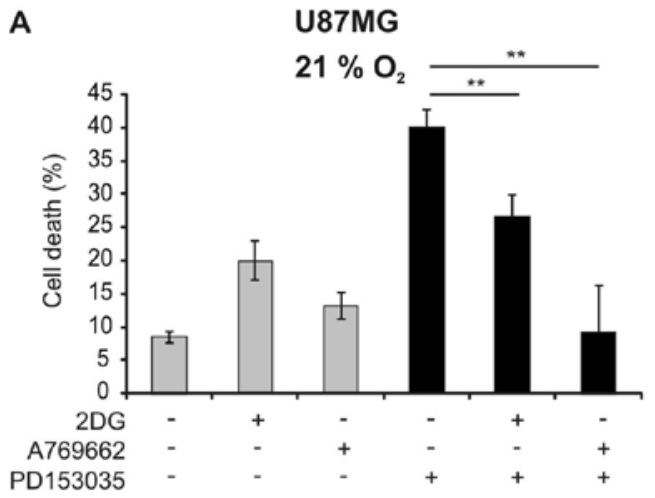

C

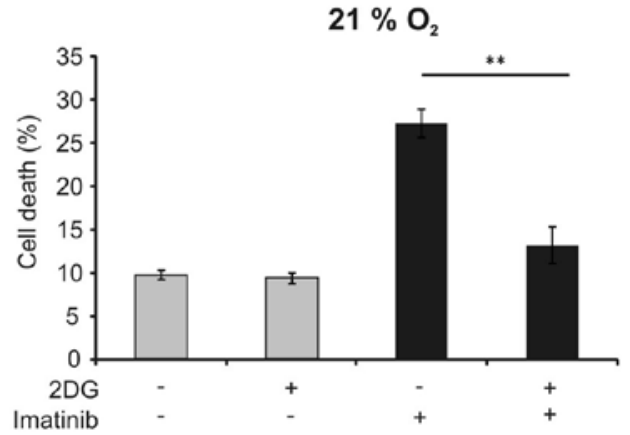

B

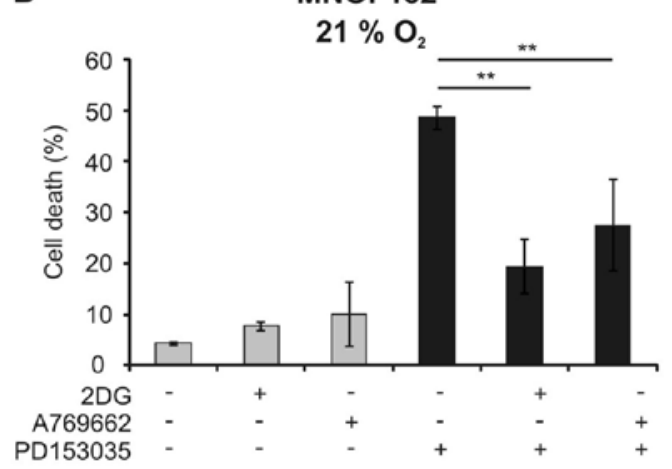

D
MDA-MB-435

$21 \% \mathrm{O}_{2}$

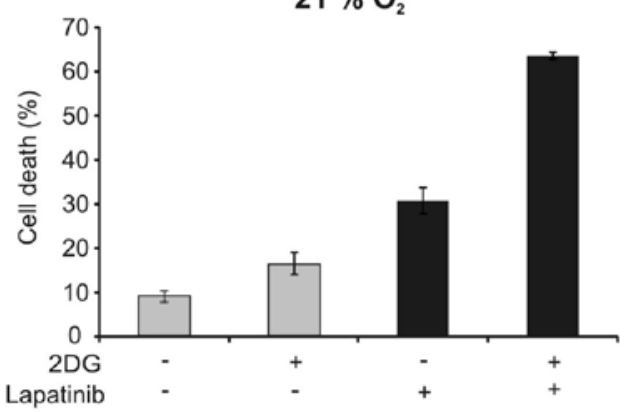

Figure 5. Antagonistic effects of AMPK activators towards tyrosine kinase inhibitors. (A and B) U87MG, MNOF132 glioma cells were control treated or exposed to 2DG, A769662 and PD153035 for $48 \mathrm{~h}$, and viability was assessed by PI-FACS. (C and D) The leukemia cell line K562 and the breast cancer cell line MDA-MB- 435 were treated as indicated for $48 \mathrm{~h}$, and cell death was assessed (means \pm SD of triplicates; ${ }^{*} \mathrm{p}<0.05,{ }^{* *} \mathrm{p}<0.01$, unpaired Student's t-test). 
A

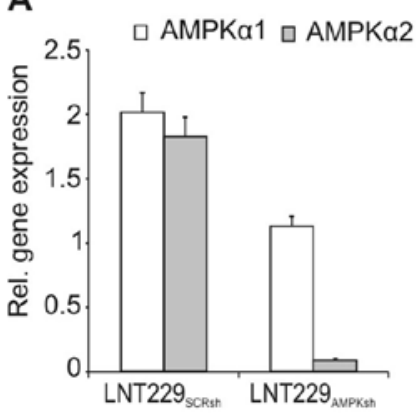

B

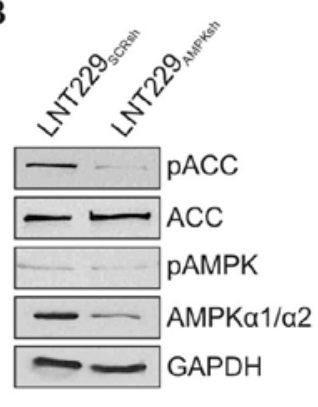

C

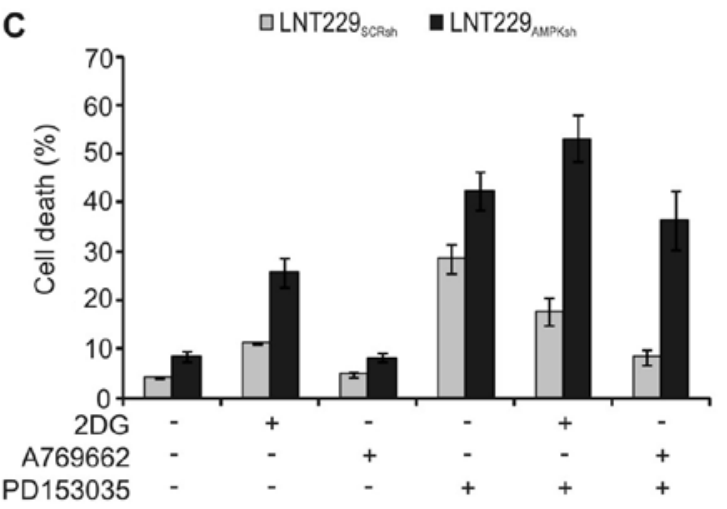

D

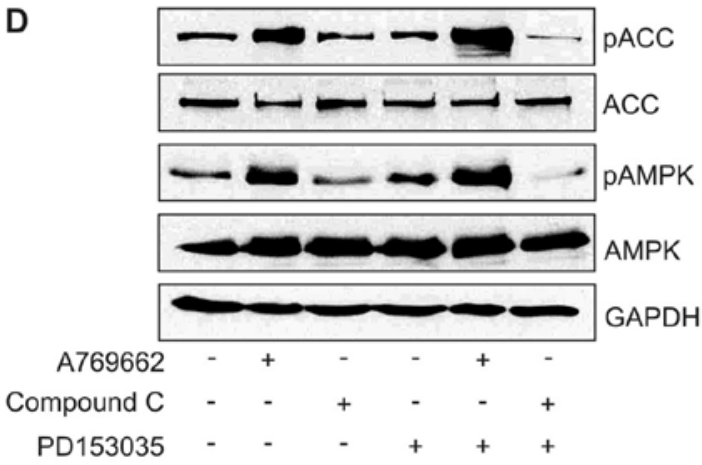

E

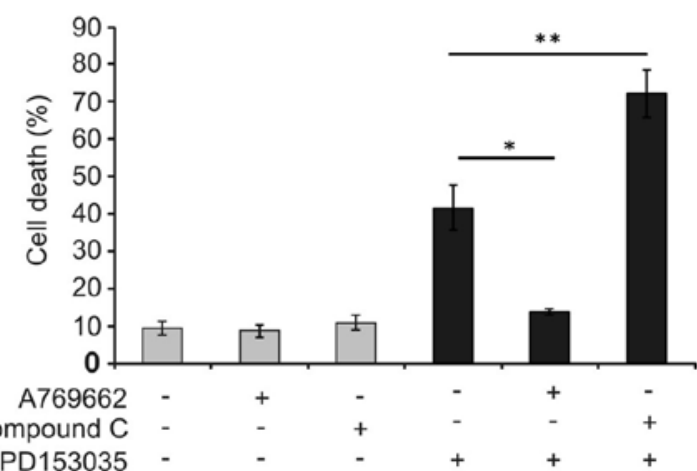

Figure 6. Knockdown and inhibition of AMPK reversed the protective effect of EGFR inhibition. (A) Expression of AMPK- $\alpha 1$ and AMPK- $\alpha 2$ was analysed in LNT-229 cells stably transfected with a control shRNA (LNT229 SCRsh) or double transfected with shRNAs against AMPK- $\alpha 1$ and $\alpha 2$ (LNT229 AMPKsh). (B) Expression of ACC, phospho-ACC, AMPK and phospho-AMPK was analysed by immunoblot analysis. GAPDH served as control. (C) LNT-229 SCRsh and AMPKsh cells were treated as indicated for $48 \mathrm{~h}$, and cell death was analysed by PI-FACS. (D) LNT-229 cells were untreated or treated with A769662 or compound $\mathrm{C}$ for $6 \mathrm{~h}$, and expression of ACC, AMPK and their phosphorylated forms was analysed by immunoblot analysis. (E) LNT-229 cells were treated as indicated for $48 \mathrm{~h}$, and cell death was analysed by PI-FACS (means \pm SD of triplicates, one experiment out of three experiments with similar results is shown; " $\mathrm{p}<0.05$, unpaired Student's t-test).

\section{Discussion}

Although EGFR is often activated in glioma, and inhibition of EGFR leads to cell death in vitro, EGFR inhibitors did not show substantial efficacy in clinical trials in glioma patients $(3,14)$. Because the microenvironment of glioma is characterised by glucose deprivation comprising levels of $\sim 0.5$ and $2 \mathrm{mM}$ (15) and severe hypoxia (16), we speculated whether these conditions might be responsible for the observed failure of tyrosine kinase inhibitors.

Whereas inhibition of EGFR led to caspase-dependent apoptosis in the presence of excess glucose (Figs. 2 and 3), cell death induction was indeed impaired by hypoxia and low glucose availability (Fig. 1). Under low glucose, phosphorylation of the cellular energy sensor AMPK was stimulated, and activation of AMPK by 2DG and A769662 reduced toxicity of EGFR inhibition (Fig. 4). In contrast, inhibition of AMPK by sh-mediated knockdown or pharmacological blockade reverted the effects of 2DG and A769662 (Fig. 5), confirming that their effect was dependent on AMPK. These results indicate that, in glioma cells, activation of AMPK might limit cytotoxicity of tyrosine-kinase inhibition. The phenomenon was not restricted to glioma cells as 2DG reduced toxicity also of imatinib in K562 cells (Fig. 5C). In contrast, 2DG did not block, but rather increased cell death induction by lapatinib in the breast cancer cell line MDA-MB-435 (Fig. 5D). The latter results are in agreement to findings in non-small cell lung cancer cells where 2DG increased cell death induction by the EGFR inhibitor afatinib (17). Therefore, the observed cytoprotective effect of AMPK activation against tyrosinekinase inhibitors seems to be cell-type specific and might be an explanation why EGFR inhibitors proved to be effective in breast and lung cancer, but not in glioma trials.

Interestingly, 2DG was more toxic in the AMPK-suppressed cells (Fig. 6C), consistent with findings in lymphoma cells (18). Similarly, synergistic cell death induction between 2DG and the AMPK inhibitor compound $\mathrm{C}$ was described in leukemia cells (19). These observations support the hypothesis that AMPK is necessary to preserve energy homeostasis during nutrient starvation in glioma. This is in agreement with the recent observation that AMPK is highly activated (20) in glioma tissue. By dissociating different molecular effects of metformin, AICAR and A769662, these authors similarly speculated that AMPK activation has pro-survival functions in nutrient starved tumors. Supporting this assumption, the eukaryotic translation elongation factor 2 kinase (eEF2K), which is activated by AMPK, has been shown to be important for resistance of tumor cells against nutrient deprivation (21). Interestingly, these authors found that expression of eEF2K is increased in glioma and associated with a worse prognosis. The finding that AMPK activators reduced glucose consumption and lactate production (Fig. 4) is congruent to the energy 
saving function of AMPK and a recently proposed model where AMPK is a central regulator of aerobic glycolysis (the 'Warburg effect') (18). The exact molecular mechanism how AMPK activation blocks EGFR inhibitor-induced toxicity remains unclear. Possible mechanisms might include the suppression of reactive oxygen species (ROS) by AMPK (22) as ROS have been proposed to be involved in the toxicity of tyrosine-kinase inhibitors (23). Additionally, metabolic alterations occurring as a result of AMPK inhibition might modulate sensitivity against tyrosine kinase inhibitors, offering novel opportunities how the efficacy of these drugs could be enhanced $(24,25)$. Together, these results suggest that activation of AMPK limits the toxicity of EGFR inhibitors in glioma cells. Determining which molecular and metabolic mechanisms mediate these cytoprotective effects of AMPK could result in strategies improving efficacy of tyrosine-kinase inhibitors.

\section{Acknowledgements}

This study was supported by grant RI2175/1-1 from the 'Deutsche Forschungsgemeinschaft' (DFG) to J.P.S. and J.R. The Dr. Senckenberg Institute of Neurooncology is supported by the Hertie foundation and the Dr. Senckenberg foundation. J.P.S. is 'Hertie Professor for Neurooncology'. S.W. was supported by grant 1748-0-0 from the interdisciplinary center for clinical research of the University of Tübingen (IZKF). M.R. received funding from the Medical Faculty, University Hospital Frankfurt (Program 'Nachwuchsforscher 2012'). J.P.S. and J.R. have served as consultants for Roche, the European distributor of bevacizumab.

\section{References}

1. Ohgaki $\mathrm{H}$ and Kleihues P: Population-based studies on incidence, survival rates, and genetic alterations in astrocytic and oligodendroglial gliomas. J Neuropathol Exp Neurol 64: 479-489, 2005.

2. Fleming TP, Saxena A, Clark WC, Robertson JT, Oldfield EH, Aaronson SA and Ali IU: Amplification and/or overexpression of platelet-derived growth factor receptors and epidermal growth factor receptor in human glial tumors. Cancer Res 52: 4550-4553, 1992.

3. van den Bent MJ, Brandes AA, Rampling R, Kouwenhoven MC, Kros JM, Carpentier AF, Clement PM, Frenay M, Campone M, Baurain JF, et al: Randomized phase II trial of erlotinib versus temozolomide or carmustine in recurrent glioblastoma: EORTC brain tumor group study 26034. J Clin Oncol 27: 1268-1274, 2009.

4. Harris AL: Hypoxia - a key regulatory factor in tumour growth. Nat Rev Cancer 2: 38-47, 2002.

5. Steinbach JP, Klumpp A, Wolburg H and Weller M: Inhibition of epidermal growth factor receptor signaling protects human malignant glioma cells from hypoxia-induced cell death. Cancer Res 64: 1575-1578, 2004.

6. Ronellenfitsch MW, Brucker DP, Burger MC, Wolking S, Tritschler F, Rieger J, Wick W, Weller M and Steinbach JP: Antagonism of the mammalian target of rapamycin selectively mediates metabolic effects of epidermal growth factor receptor inhibition and protects human malignant glioma cells from hypoxia-induced cell death. Brain 132: 1509-1522, 2009.

7. Düvel K, Yecies JL, Menon S, Raman P, Lipovsky AI, Souza AL, Triantafellow E, Ma Q, Gorski R, Cleaver S, et al: Activation of a metabolic gene regulatory network downstream of mTOR complex 1. Mol Cell 39: 171-183, 2010.

8. de Groot JF, Fuller G, Kumar AJ, Piao Y, Eterovic K, Ji Y and Conrad CA: Tumor invasion after treatment of glioblastoma with bevacizumab: Radiographic and pathologic correlation in humans and mice. Neuro-oncol 12: 233-242, 2010.
9. Steinbach JP, Supra P, Huang H-JS, Cavenee WK and Weller M: CD95-mediated apoptosis of human glioma cells: Modulation by epidermal growth factor receptor activity. Brain Pathol 12: 12-20, 2002.

10. Steinbach JP, Wolburg H, Klumpp A, Probst H and Weller M: Hypoxia-induced cell death in human malignant glioma cells: Energy deprivation promotes decoupling of mitochondrial cytochrome c release from caspase processing and necrotic cell death. Cell Death Differ 10: 823-832, 2003

11. Fischer S, Ronellenfitsch MW, Thiepold A-L, Harter PN, Reichert S, Kögel D, Paschke R, Mittelbronn M, Weller M, Steinbach JP, et al: Hypoxia enhances the antiglioma cytotoxicity of B10, a glycosylated derivative of betulinic acid. PLoS One 9: e94921, 2014.

12. Wanka C, Brucker DP, Bähr O, Ronellenfitsch M, Weller M, Steinbach JP and Rieger J: Synthesis of cytochrome C oxidase 2: A p53-dependent metabolic regulator that promotes respiratory function and protects glioma and colon cancer cells from hypoxia-induced cell death. Oncogene 31: 3764-3776, 2012.

13. Wanka C, Steinbach JP and Rieger J: Tp53-induced glycolysis and apoptosis regulator (TIGAR) protects glioma cells from starvation-induced cell death by up-regulating respiration and improving cellular redox homeostasis. J Biol Chem 287: 33436-33446, 2012.

14. Peereboom DM, Shepard DR, Ahluwalia MS, Brewer CJ, Agarwal N, Stevens GH, Suh JH, Toms SA, Vogelbaum MA Weil RJ, et al: Phase II trial of erlotinib with temozolomide and radiation in patients with newly diagnosed glioblastoma multiforme. J Neurooncol 98: 93-99, 2010.

15. Marcus HJ, Carpenter KLH, Price SJ and Hutchinson PJ: In vivo assessment of high-grade glioma biochemistry using microdialysis: A study of energy-related molecules, growth factors and cytokines. J Neurooncol 97: 11-23, 2010.

16. Collingridge DR, Piepmeier JM, Rockwell S and Knisely JP: Polarographic measurements of oxygen tension in human glioma and surrounding peritumoural brain tissue. Radiother Oncol 53: 127-131, 1999.

17. Kim SM, Yun MR, Hong YK, Solca F, Kim J-H, Kim H-J and Cho BC: Glycolysis inhibition sensitizes non-small cell lung cancer with T790M mutation to irreversible EGFR inhibitors via translational suppression of Mcl-1 by AMPK activation. Mol Cancer Ther 12: 2145-2156, 2013.

18. Faubert B, Boily G, Izreig S, Griss T, Samborska B, Dong Z, Dupuy F, Chambers C, Fuerth BJ, Viollet B, et al: AMPK is a negative regulator of the Warburg effect and suppresses tumor growth in vivo. Cell Metab 17: 113-124, 2013.

19. Miwa H, Shikami M, Goto M, Mizuno S, Takahashi M, Tsunekawa-Imai N, Ishikawa T, Mizutani M, Horio T, Gotou M, et al: Leukemia cells demonstrate a different metabolic perturbation provoked by 2-deoxyglucose. Oncol Rep 29: 2053-2057, 2013.

20. Liu X, Chhipa RR, Pooya S, Wortman M, Yachyshin S, Chow LM, Kumar A, Zhou X, Sun Y, Quinn B, et al: Discrete mechanisms of mTOR and cell cycle regulation by AMPK agonists independent of AMPK. Proc Natl Acad Sci USA 111: E435-E444, 2014.

21. Leprivier G, Remke M, Rotblat B, Dubuc A, Mateo AR, Kool M, Agnihotri S, El-Naggar A, Yu B, Somasekharan SP, et al: The eEF2 kinase confers resistance to nutrient deprivation by blocking translation elongation. Cell 153: 1064-1079, 2013.

22. Jeon S-M, Chandel NS and Hay N: AMPK regulates NADPH homeostasis to promote tumour cell survival during energy stress. Nature 485: 661-665, 2012.

23. Qian X, Li J, Ding J, Wang Z, Zhang W and Hu G: Erlotinib activates mitochondrial death pathways related to the production of reactive oxygen species in the human non-small cell lung cancer cell line A549. Clin Exp Pharmacol Physiol 36: 487-494, 2009.

24. Zhao F, Mancuso A, Bui TV, Tong X, Gruber JJ, Swider CR, Sanchez PV, Lum JJ, Sayed N, Melo JV, et al: Imatinib resistance associated with BCR-ABL upregulation is dependent on HIF-1alpha-induced metabolic reprograming. Oncogene 29: 2962-2972, 2010.

25. De Rosa V, Iommelli F, Monti M, Fonti R, Votta G, Stoppelli MP and Del Vecchio S: Reversal of Warburg effect and reactivation of oxidative phosphorylation by differential inhibition of EGFR signaling pathways in non-small cell lung cancer. Clin Cancer Res 21: 5110-5120, 2015. 